\title{
Are the Therapeutic Effects of Homeopathy Attributed to the Consultation, the Homeopathic Remedy, or Both? A Protocol for a Future Exploratory Feasibility Trial in Patients with Rheumatoid Arthritis
}

\author{
SARAH BRIEN, Ph.D., ${ }^{1}$ LAURIE LACHANCE, Ph.D., ${ }^{2}$ and GEORGE T. LEWITH, M.D., F.R.C.P. ${ }^{1}$
}

\begin{abstract}
Systematic reviews suggest that homeopathy has a greater effect than placebo, however, the mechanisms of its action are unknown. The clinical effects of homeopathy could be attributed to the specific effects of the remedy and/or to the contextual effects of the consultation process; these factors have not been critically evaluated. We have developed a model that attempt to separate the consultation effects from the specific effects because of the remedy. We propose to investigate this design in a chronic condition, rheumatoid arthritis, for which previous research has evaluated both classical and complex homeopathic interventions. The following protocol describes the study design. The aims of this exploratory trial are to assess the feasibility of the study design, identify effect sizes of the consultation, the complex treatment, and the individualized remedy, as well as possibly efficacy, for the two types of homeopathic treatment and the homeopathic consultation. Data collection will take place in 2004 .
\end{abstract}

\section{INTRODUCTION}

$\mathbf{H}$ omeopathy is contentious. Surveys confirm that patients use and continue to use homeopathy (Eisenberg et al., 1998), and feel satisfied with their treatment (Richardson, 2001). A meta-analysis (Linde et al., 1997) and three systematic reviews (Cucherat et al., 2000; Hill and Doyon, 1990; Kleijnen et al., 1991) suggest that in "good" quality trials, homeopathy has a significantly greater effect than placebo (Hill and Doyon, 1990; Kleijnen et al., 1991; Linde et al., 1997) although the strength of the effect is disputable (Cucherat et al., 2000) and engenders much debate. However these positive clinical effects are still largely unexplained (Linde et al., 1997) because currently no plausible mechanism to explain its action exists. In addition, previous randomized controlled trials show that therapeutic improvements are observed in both verum and placebo groups
(Linde et al., 1997). This has raised questions about where the effects of homeopathy lie: is it the material and chemical interactions caused by the homeopathic remedy or the nonspecific ("contextual") effects of the consultation, or both?

Contextual factors include those that are not the result of the active components of the treatment but are inherent within the treatment "package," (Di Blasi et al., 2001; Hyland, 2003; Moerman and Jonas 2002; Walach, 2003) and can include a myriad of factors related to the practitioner (e.g. the practitioners' manner and attention to their patients, their expectations, etc.), the patient (expectations of outcome, attitudes, hope, philosophical belief in treatment, etc.), and the therapeutic relationship as well as the characteristics of the setting, for example, the credibility of the treatment. Current trends have shifted toward understanding those factors such as the therapeutic relationship and patient-

\footnotetext{
${ }^{1}$ Complementary Medicine Research Unit, University of Southampton, Primary Medical Care, Aldermoor Health Centre, Southampton, Hampshire, UK.

${ }^{2}$ School of Public Health, The University of Michigan, Ann Arbor, MI.
} 
centered factors (e.g., empathy, empowerment, etc; Di Blasi et al., 2001) that may mediate the apparent success of the homeopathic process.

This current study propose a conceptual model to separate out and identify the effect sizes of both the specific and nonspecific aspects of the homeopathic process. We propose to test this model in the chronic condition rheumatoid arthritis (RA) in which there is both evidence and a rationale to use both classical and complex homeopathic approaches. A meta-analysis of three of the four randomized controlled trials (three trials used individualized homeopathy [Andrade et al., 1991; Fisher and Scott, 2001; Gibson et al., 1980]) and one used complex homeopathy that was reported in the literature twice (Kohler, 1991; Wiesenauer and Gaus, 1991) identified an odds ratio of 2.0 in favor of homeopathy although only the study that used complex homeopathy showed a clear statistically significant result (Kohler, 1991; Wiesenauer and Gaus, 1991). Therefore, based in the context of this disease, this protocol proposes a conceptual model to answer the principal question of the study: "Can the effects of the homeopathic consultation be separated and measured separately from the homeopathic remedy?"

This will not be a definitive study. The aims of this protocol will be to assess the feasibility of the study design, to identify the consultation process and separate it from the homeopathic remedy, and to identify the following effect sizes: (1) the placebo effect, with and without the consultation, (2) the effect of the consultation, (3) the effect of the individual remedy, (4) the effect of the homeopathic complex, and (5) the difference in effects between complex homeopathy and individual homeopathy. Other additional patient centered aims were identified.

\section{MATERIALS AND METHODS}

The trial design is a placebo-controlled, randomized double-blinded exploratory pilot trial will be conducted at Well- come Trust Clinical Research Facility, Southampton General Hospital.

Patients with relatively stable RA disease will be recruited from teaching hospitals in the southern part of England. Inclusion criteria will be as follows: between 18-75 years of age, diagnosed with RA for more than 2 years (based on American College of Rheumatology [ACR] Guidelines; Arnett et al., 1988); on stable medication for longer than 2 months. Exclusion criteria includes patients with severe comorbidities, those taking immunosuppressant drugs or biologic disease-modifying antirheumatic drugs (i.e., antitumor-necrosis factor [TNF]); those classified as functional status class IV in the ACR criteria; use of homeopathy less than 3 months; and pregnancy or breastfeeding. There will be 20 per arm.

Patients will be randomized into five groups as shown in Figure 1 to investigate the effects of homeopathy with and without a consultation. Three of the groups received a consultation (groups 1, 2, and 3) and two groups (groups 4 and 5) will receive no consultation. The consultation group patients will be randomized to receive either individualized treatment (group 1), a complex designed for RA (group 2), or placebo (group 3).

Those subjects allocated to groups 4 and 5 will receive either the complex or placebo. The study design allowed for the following comparisons to be investigated:

- The effect of the consultation can be assessed by comparing the consultation and nonconsultation groups (i.e., group 2 versus group 4), and also from group 3 versus group 5.

- The effect of the complex treatment can be assessed by comparing groups 2 versus 3 and, additionally, group 4 versus group 5 .

- The effect of the individualized remedy can be assessed by comparing groups 1 and 3 .

- The difference in effect between the individualized treatment and the complex can be determined by comparing group 1 and group 2 .

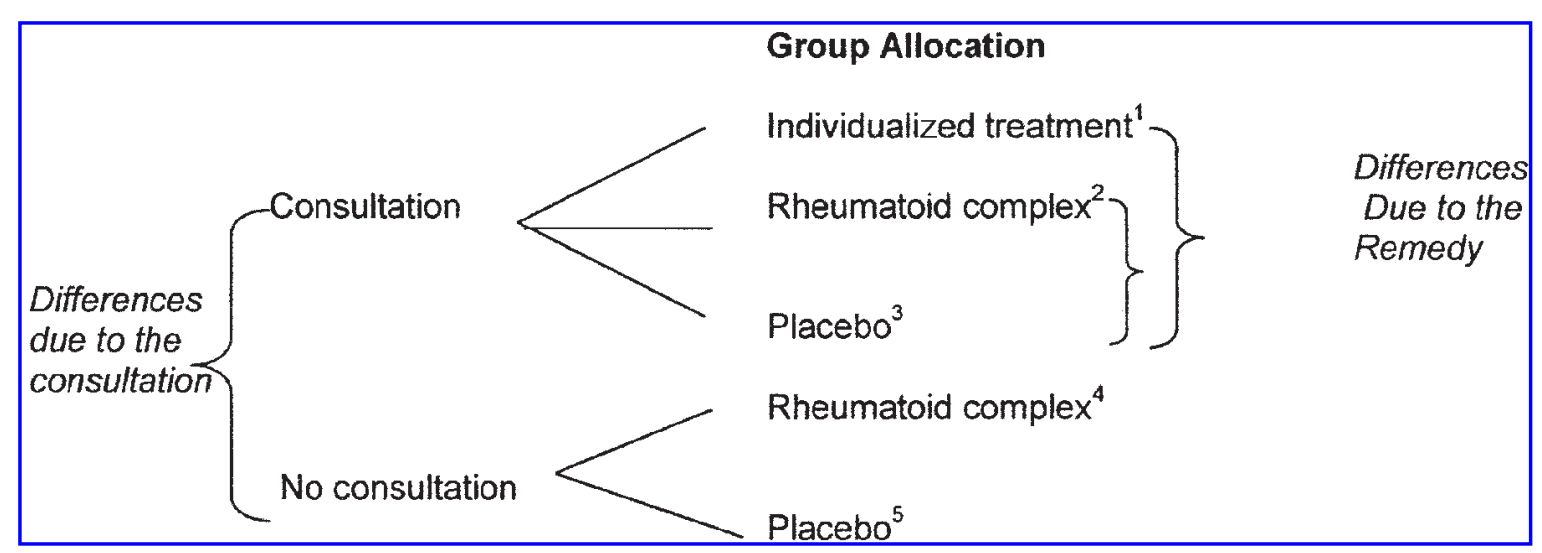

FIG. 1. Study design showing randomization scheme. Numbers represent study groups as described in the text. 


\section{Treatment}

Patients will be allocated to one of the five study arms, as described in Figure 1. Each patient will be enrolled in the study for 10 months (i.e., 1 month baseline, 6 months of treatment, and 3 months of follow-up). After baseline assessment all patients will attend a standardized monthly clinic visit during the treatment phase where outcome measures will be recorded by the study nurse. Subjects who are randomized to consultation will also receive a homeopathic consultation. The homeopaths, blinded to treatment allocation, will prescribe from the whole repertoire but remain restricted to a standardized dosing regimes to maintain blinding. Homeopathic prescriptions for each patient who receive consultations will be faxed to an independent pharmacy where the appropriate prescription (i.e., a classically prescribed remedy for group 1, a complex for group 2, and a placebo for group 3) will be sent to the patients. Patients will complete weekly diaries between clinic visits. Subjects will be followed up 3 months after the treatment phase. Strategies to minimize drop-out rates will be used.

\section{Trial medication}

The independent pharmacy will send all patients their study medication after each clinic visit during the treatment phase. This will include 1 bottle of tablet medication (which will contain either classical remedy or placebo as appropriate, to be taken one tablet twice per day) and a bottle of liquid (which will contain the homeopathic complex or placebo as appropriate with a dosing regimen of 20 drops twice per day). The complex remedy is a commercial preparation (Dreluso, Hessisch Oldendorf, Germany) containing Rhus Tox D4, Bryonia cretica D4, Stychnos nux vomica D4, Berberis vulgaris D4, Ledum palustre D4 in a 20-mL solution that has been used in previous trials (Kohler, 1991; Wiesenauer and Gaus, 1991). The placebo complex remedy will contain the same as the verum minus the homeopathic remedies. Individualized remedies will be prescribed according to the consultation from the whole repertoire and in any appropriate dose but on standard dosing frequency. The medication can be prepared as prescribed constitutional (i.e., tablets 1 to 3 would be the constitutional remedy prescribed) followed by dummy or acute remedy (for tablets 4 to end). For subjects who receive placebo, all tablets will contain placebo.

\section{OUTCOME ASSESSMENT}

The primary outcome measures will be: (1) whether the subjects achieve a $20 \%$ improvement (binary variable) in global RA symptoms based on the outcome measures from the Outcome Measures for Arthritis Clinical Trials (OMERACT; Fries et al., 1980), which include assessment of joint pain/tenderness, joint swelling, visual analogue scale (VAS) pain, acute phase reactants, functional status, and pa- tient and physician global assessment; and (2) weekly VAS pain scores. There will be a number of secondary outcomes including individual assessment of OMERACT outcomes, quality of life assessment, and global assessments. A range of covariates will be investigated as possible predictors of treatment outcome (e.g., patient/physician's expectations, attitudes about CAM, and for subjects who are allocated to consultation patient perceived empathy, enablement, and sense of emotional meaning attributable to the process).

\section{Data analysis}

The data will be analyzed on an intention-to-treat basis. The binary primary outcome variable will be evaluated using logistic regression analysis, adjusted for baseline and other confounding factors. The continuous primary outcome measure will be assessed by repeated measures analysis of variance (ANOVA). Pair-wise comparisons will be made between those receiving a homeopathic remedy (complex) versus placebo in consultation and nonconsultation groups.

\section{RESULTS}

Data collection will commence 2004. Part funding has been secured from the Samueli Institute, Corona del Mar, CA.

\section{CONCLUSION}

It is not anticipated that the power of the study will be adequate to identify small group differences, and the design is based on the assumption that the effects of case taking, the remedy and other effects that contribute to the study effect are additive. However, this study will identify whether the model proposed to separate the effects of the homeopathic consultation from the remedy is feasible. In addition this study will provide further evidence for the effect of homeopathy in rheumatoid arthritis. If it is successful, this investigative model may have broad relevance to both CAM and conventional medicine.

\section{ACKNOWLEDGMENT}

Dr. Sarah Brien is funded by Hospital Savings Association and Dr. George Lewith by Laing Foundation. The Samueli Institute, Corona del Mar, CA, will provide part funding for the study.

\section{REFERENCES}

Andrade L, Ferraz MB, Atra E, Castro A, Silva MSM. A randomized controlled trial to evaluate the effectiveness of home- 
opathy in rheumatoid arthritis. Scand J Rheumatol 1991;20: 204-208.

Arnett FC, Edworthy SM, Bloch DA, McShane DJ, Fries JF, Cooper NS, Healey LA, Kaplan SR, Liang MH, Luthra HS. The American Rheumatism Association 1987 revised criteria for the classification of rheumatoid arthritis. Arthritis Rheum 1988;31: 315-324.

Cucherat M, Haugh MC, Gooch M, Boissel JP. Evidence of clinical efficacy of homeopathy. A meta-analysis of clinical trials. Eur J Clinical Pharmacol 2000;56:27-33.

Di Blasi Z, Harekness E, Ernst E, Georgiou A, Kleijnen J, Influence of context effects on health outcomes: A systematic review. Lancet 2001;357:757-762.

Eisenberg DM, Davis RB Ettner SL, Appel S, Wilkes S, Van Rompney, Kessler RC. Trends in Alternative Medicine Use in the United States, 1990-1997. JAMA 1998;280:1569-1575.

Fisher P, Scott D. A randomised controlled trial of homeopathy in rheumatoid arthritis. Rheumatology 2001;40:1052-1055.

Fries JF, Spitz P, Kraines RG, Holman HR. Measurement of patient outcome in arthritis. Arthritis Rheum 1980;23:137-145.

Gibson RG, Gibson S, MacNeill AD, Watson W. Homeopathic therapy in rheumatoid arthritis: Evaluation by double-blind clinical therapeutic trial. Br J Clin Pharmacol 1980;9:453-459.

Hill C; Doyon F. Review of randomized trials of homeopathy. Rev Epidemo Dante Publique \1990:38:139-147.

Hyland M. Using the placebo response in clinical practice. Clin Med 2003;3:347-350.

Kleijnen J, Knipschild P, ter Riet G. Clinical trials of homeopathy. B Med J 1991;302:316-322.

Köhler T: Wirksamkeitsnachweis eines Homöopathikums bei chro- nischer Polyarthritis eine randomisierte doppelblindstudie bei niedergelassenen Ärzten. Der Kassenarzt 1991;13:48-52.

Linde K, Clausius N, Ramirez G, Melchart D, Eitel F, Hedges LV, Jonas WB. Are the clinical effects of homeopathy placebo effects? A meta-analysis of placebo-controlled trials. Lancet 1997;350:834-843.

Moerman D, Jonas W. Deconstructing the placebo effect and finding the meaning response. Ann Intern Med 2002;136:471-476.

Richardson WR. Patient benefit survey: Liverpool Regional Department of Homeopathic Medicine. Br Homeopath J 2001; 90:158-162.

Walach H. Placebo and placebo effects-A concise review. Focus Altern and Complement Ther 2003;8:178-187.

Wiesenauer M, Gaus W: Wirksamkeitsnachweis eines Homöopathikums bei chronischer Polyarthritis. Eine randomisierte doppelblindstudie bei niedergelassenen Ärzten. Aktuelle Rheumatologie 1991;16:1-9.

Address reprint requests to: Sarah Brien, Ph.D. Complementary Medicine Research Unit University of Southampton Primary Medical Care Aldermoor Health Centre Aldermoor Close Southampton, Hampshire, SO16 5ST United Kingdom

E-mail: s.brien@soton.ac.uk 
This article has been cited by:

1. Jennifer Jacobs , Anna-Leila Williams , Christine Girard, Valentine Yanchou Njike , David Katz . 2005. Homeopathy for Attention-Deficit/Hyperactivity Disorder: A Pilot Randomized-Controlled TrialHomeopathy for Attention-Deficit/Hyperactivity Disorder: A Pilot Randomized-Controlled Trial. The Journal of Alternative and Complementary Medicine 11:5, 799-806. [Abstract] [PDF] [PDF Plus] 